\title{
La interculturalidad del español: corrección y adecuación de saludos y direcciones en la evaluación de profesores nativos
}

\author{
The interculturality of Spanish: correctness \\ and adequacy of greetings \\ and addresses in the evaluation for native teachers
}

\author{
María Cecilia Ainciburu \\ Universidad Nebrija (España) / Università degli Studi di Siena (Italia) \\ caincibu@nebrija.es / ainciburu@unisi.it \\ David Rodríguez Velasco \\ Queen's University of Belfast (Reino Unido) / Universidad Nebrija (España) \\ d.rodriguez-velasco@qub.ac.uk / drodriguezv1@alumnos.nebrija.es
}

\begin{abstract}
This research aims to investigate the presence in textbooks of varied material in terms of geolectal equivalents and its perception by teachers of Spanish as a foreign language. In order to examine potential differences in their approach, a pedagogical framework-based task was designed to study a corpus of 100 ELE teachers' evaluations (50 from Colombia and 50 from Spain) and examine their consciousness and dissimilarities regarding pragmatic correctness when expressing addresses and greeting headings in electronic messages. The results show that native teachers tend to consider as correct the stimuli of peninsular Spanish or of their own variety, while those representing different geolectal variations of Spanish tend to score very low, even being considered as inter or intralinguistic error.
\end{abstract}

Keywords: greetings, addresses, interculturality, Spanish teachers, geolectal variations 


\section{INTRODUCCIÓN}

Cuando hablamos de variación en las funciones lingüísticas a partir de las cuales estructuramos la enseñanza del español como lengua extranjera (en adelante, ELE), tenemos en cuenta dos tipos de fenómeno: por un lado, la existencia de formas equivalentes de expresión, de naturaleza geolectal en la lengua meta y, por otro, la posibilidad de que el estudiante extranjero produzca estructuras idiosincráticas, no esperadas y a veces poco adecuadas en el intercambio con el nativo. Así, por ejemplo, escuchamos Buen día o Buenos días en diferentes lugares en los que se habla español y Buenas días en boca de algún extranjero. En muchos casos se supone que la falta de adecuación se origina casi siempre por interferencia de la lengua materna y de la cultura de proveniencia en la producción en lengua extranjera (Li \& Chen, 2016). De hecho, esto no explica la producción de Buenas días (error intralingüístico) ni la posible opción por una de las correctas. Fuera del ámbito de las investigaciones pragmáticas se suele intentar redimensionar la hipótesis de transferencia negativa como mecanismo explicativo más difundido (Alba Quiñones, 2009).

La investigación aplicada ha mostrado que no es posible hablar de una sola cultura del español o de una forma única de cortesía. Nuestra hipótesis es que los manuales ELE tienden a desestimar estas formas de variación alternativas o a relegarlas a piezas léxicas. Es muy común que los manuales presenten alternativas geosinonímicas como camión, guagua, micro, autobús, por ejemplo en un manual ELE A2, pero es más difícil hallar una explicación que aprecie la variación entre Buen día / Buenos días que ocurre incluso dentro de la Península. Teniendo en cuenta estas deficiencias, el docente debería percatarse de este problema, poseer un conocimiento amplio de las variantes del español y de los usos pragmáticos de la lengua que enseñan y aplicarlo mediante el apoyo tanto del contenido de sus manuales como de materiales complementarios (por ejemplo, de la web). En este sentido, aunque ser profesor nativo debería representar una ventaja inicial en términos de formación y de comportamiento como evaluador (Andión Herrera, 2019) porque el conocimiento de las variedades debería ser más profundo, parte de las investigaciones muestran que los elementos pragmáticos suelen ser más evidentes para los docentes no nativos (De la Fuente Cobas \& Wei, 2020).

Esta investigación indaga acerca del criterio con el que los docentes nativos juzgan la adecuación de una fórmula léxica en un contexto de uso. Ha sido financiada por el Ministerio de Ciencia, Innovación y Universidades del Reino de España (Proyecto "Emoción, memoria, identidad lingüística y aculturación emocional: su influencia en el aprendizaje de español como lengua de migración"; FFI2017-83166-C2-2-R). Por razones de representatividad, se han elegido dos funciones: las direcciones postales y el saludo en un correo electrónico al profesor. Estas dos funciones se caracterizan por ser fuertemente formulaicas y entrar en los sílabos de enseñanza de lengua, lo que debería predecir su presencia en los manuales de ELE básicos. Además, y dado que 
este es un punto fundamental en una óptica de variación de la lengua, tienen equivalentes geolectales: igual que no se saluda al profesor del mismo modo en Madrid o en Buenos Aires, tampoco se localiza igual un inmueble en estas ciudades.

\section{LAS DIRECCIONES}

La planificación didáctica secuencial de las "direcciones postales" en el sílabo ELE se encuentra en la sección "Datos personales" y en el apartado "3.2.1 Dirección" del Plan curricular del Instituto Cervantes (en adelante, PCIC). En el nivel A1 se integran las unidades léxicas necesarias (dirección, correo electrónico, calle, avenida, paseo, plaza, piso, número, código postal, casa, habitación, país, ciudad, pueblo, vivir), en los niveles siguientes, no se mencionan otros y los que se introducen en B2 (periferia, casco antiguo, centro histórico) difícilmente se integran en una dirección postal.

En el PCIC esa función no está presente entre los saberes culturales, posiblemente porque existe una objetividad de las direcciones postales que las hace independientes de la composición libre. Se trata de fórmulas en las que el grado de libertad es mínimo y es posible que su lugar de referencia sean las Nociones específicas. La única variación estudiada allí es la de registro, ya que se ejemplifica "la variedad centro-norte peninsular española y en las características idiosincrásicas de España" (PCIC: Nociones específicas. Introducción), lo que excluye la geosinonimia. Creemos que la correcta interpretación de una dirección postal incluye otros saberes lingüísticos (rol de las mayúsculas, significado de las abreviaturas) que pueden aumentar su nivel de dificultad.

Es posible que la acción misma de escribir una dirección en un sobre sea demodé, pero la hemos elegido porque es una función básica y como tal, una cartilla de tornasol en los manuales de ELE. Los manuales ELE editados por tres editoriales escogidas al azar (SGEL, Difusión y Edinumen) no contienen casi direcciones postales (53 manuales revisados, seis ocurrencias). En ámbito europeo la situación no es idéntica, ya que algunos manuales redactados para estudiantes italianos o alemanes contienen direcciones postales hispanoamericanas. Resulta llamativo que un manual diseñado para el público universitario alemán (Ainciburu et al., 2009) contuviera una actividad de reflexión intercultural donde se veían sobres con direcciones de diferentes países de habla española (Anexo 1) y que la haya perdido cuando se republicó en España bajo la guía de otro editor (Ainciburu et al., 2011).

Gavarrete \& Albanese (2018) proponen una mediación en el aula nativa, a partir del estudio de las direcciones postales y las indicaciones para ubicar un lugar con la intención de concretizar dos conceptos matemáticos: el plano cartesiano y la estimación de medidas de longitud. Se contraponen las direcciones postales de ciudades planificadas por manzanas frente a las de entornos rurales. El objetivo explícito de las actividades propuestas es "contextualizar el proceso de enseñanza y aprendizaje de un tema concreto de la matemática escolar teniendo en cuenta el legado cultural y la for- 
ma idiosincrática de dar las direcciones en diferentes países" (Gavarrete \& Albanese, 2018 , p. 27). No hallamos propuestas similares para ELE, aunque resulta claro que la forma de escribir las direcciones postales es un rasgo típico de cada cultura y los conocimientos conceptuales (la numeración de los edificios y casas, la diferencia entre "calle", "avenida", "costanera", entre otras) y más detalladamente lingüístico (el valor de las abreviaturas) requieren para los nativos y para los extranjeros una exposición al input directo o de realia que los contengan.

\section{LOS SALUDOS}

En el PCIC, los saludos se incluyen como "Funciones" y también como "Saberes culturales", lo cual representa un avance respecto a las direcciones postales. En la secuenciación la inmisión de esta función por nivel en la lengua escrita es: Querido / $a+$ nombre de pila (A2), Señor / Señora + apellido, Muy Sr. Mío/Señores, Estimado + nombre de pila, Estimado Sr. + apellido (B1) y las formas de tratamiento como Ilmos. Sres. (C2). Así, un estudiante ELE de nivel B1 debería poseer los recursos necesarios para dirigirse por escrito a su profesor utilizando un tratamiento formal o informal intencionalmente.

Las investigaciones sobre la fase de apertura de las "cartas al docente" de estudiantes no nativos (Economidou-Kogetsidis, 2011; Li \& Chen, 2016; Codina Espurz \& Salazar Campillo, 2019) identifican como una parte de la macroestructura textual el momento de la apertura (opening) donde se produce el saludo con el uso de una fórmula. El estudio de Economidou-Kogetsidis (2011) las releva siguiendo una lógica de composición formulaica con tres submovimientos prevalentes: (1) el uso de Dear (con nombre, apellido, título académico correcto o incorrecto y sus posibles combinaciones de dos o tres componentes) o (2) su omisión, sumado a la posibilidad de (3) uso de saludos (greetings) como Hi o Hello, que sustituyen el Dear en combinaciones similares (Economidou-Kogetsidis, 2011, p. 12).

Estas investigaciones resultan útiles para describir el desarrollo de la interlengua de estudiantes extranjeros, pero se basan en la hipótesis de interferencia atribuida a la falta de presentación sistemática de los contenidos pragmáticos en el aula y en los libros de texto (Economidou-Kogetsidis, 2011; Níkleva \& Núñez Delgado, 2013; Codina Espurz \& Salazar Campillo, 2019). En términos lingüísticos, la situación específica del español es diferente a la del inglés. Asumir la descripción de estos autores conlleva dos problemas metodológicos: en primer lugar, las combinaciones no se presentan en un corpus accesible ni con un análisis cuantitativo que dé cuenta cabal del fenómeno y, en segundo lugar, no se ligan directamente a su adecuación pragmática, ya que desde el punto de vista del registro "Dear Noam" no tiene el mismo impacto que "Dear Chomsky". En ambos casos se trata de dos combinaciones correctas 
(nombre o apellido), pero de niveles de tratamiento diferente. Además, esa misma combinación y sus posibilidades de inadecuación pragmática se hacen más complejas en español, porque los posibles usos de "Estimado" o "Querido" no son equivalentes entre sí, aunque sean buenas traducciones de "Dear".

La investigación de Codina Espurz \& Salazar Campillo (2019) muestra que el 90\% de los estudiantes españoles que escribieron correos electrónicos en cursos de inglés como lengua extranjera prefirieron utilizar la fórmula de apertura "Saludo + Nombre de pila" frente a un mínimo 10\% que empleó el saludo simple a la hora de iniciar sus correos electrónicos en inglés. En ese sentido, presenta una situación menos variada que la de Economidou-Kogetsidis (2011). Las autoras verifican la existencia de una limitación en la competencia pragmática de estos estudiantes, restringida a tan solo dos fórmulas: "Saludo" o "Saludo + Nombre de pila" y la nula presencia del título académico para dirigirse al docente, lo que reflejaría una falta de conciencia sobre las relaciones y la distancia sociales en los correos electrónicos estudiantiles. Se concluye que estos resultados reflejan la necesidad de una instrucción de carácter sociopragmático en el uso de las fórmulas de saludo con el empleo de un tono adecuado y deferencial. Según nuestro parecer, hay una pieza que se echa en falta en el análisis: la posibilidad de la influencia de un "efecto cercanía" dado por la cultura de inmersión de profesores y estudiantes que, si bien se comunican académicamente en inglés (porque los cursos son de educación bilingüe y así se les requiere), están inmersos en una cultura universitaria, la de la Universitat Jaume I, en la que profesores y estudiantes se comunican en español y se dan de tú y se llaman por su nombre.

La tesis doctoral de Robles Garrote (2017) nos aproxima mejor al problema de las combinatorias en español y a un corpus comparativo, ya que en los anexos contiene las muestras analizadas. La autora focaliza el problema del acto de habla del "pedido" en estudiantes italianos de ELE y llega a similares conclusiones sobre el orden de interferencia lingüística y la falta de recursos en los manuales. El capítulo "Resultados" presenta la macroestructura del correo electrónico y el contraste entre producción nativa y no nativa. El análisis cualitativo se basa en el uso de las estrategias presentadas en la taxonomia de Brown \& Levinson (1978), por lo que las estrategias utilizadas en el submovimiento de apertura se elencan así: mostrar (1) deferencia en el saludo formal (cortesía mitigadora), (2) cercanía hacia el destinatario con el nombre de pila o (3) con el tuteo (ambas formas de cortesía valorizante).

Resulta claro que se trata de estrategias que la investigadora considera como valorizantes o mitigadoras desde el punto de vista de la intención del hablante. Muy diferente será entender si el docente-receptor las considerará tales, sobre todo si las culturas académicas del español no son homogéneas. En algunos países, llamar al profesor por nombre y no por apellido es la norma, mientras que en otros es un acercamiento que se percibe como indebido. Las muestras nativas de Robles Garrote (2017) son peninsulares y esto introduce un sesgo, dado que la práctica académica del tuteo entre 
profesor y estudiante parece mayormente difundida en la Península. En el corpus presentado por la autora hay muestras que contienen aperturas como "Buenos días, José" que, aunque se suponen "valorizantes" en el análisis, se percibirían como inadecuadas y lograrían un efecto opuesto en algunos contextos universitarios americanos.

En el caso de las investigaciones reseñadas, tanto en inglés como en español, el criterio de adecuación no necesariamente se valida o discute, como es claro en nuestra objeción a Codina Espurz \& Salazar Campillo (2019). En la mayor parte de los casos solo el investigador o los autores del artículo han decidido qué es adecuado y qué no. Esto da la errada certeza de que esos actos de habla son adecuados a contextos similares sin que exista un rasgo cultural de diferenciación. Sabemos, en cambio, que el tuteo difundido en las universidades españolas resulta una práctica no sostenida en otros contextos universitarios del continente americano.

\section{ESTE ESTUDIO}

La pregunta de investigación que vertebró la investigación primaria fue:

- Dada una serie de estímulos de "direcciones" y "saludos al profesor" que presentan variaciones pragmáticas (de registro o geolectales) en evaluaciones ELE simuladas, ¿considerarán los docentes que los estímulos de las variedades que no son la propia son adecuados en el uso contextual (registro) o los considerarán incorrectos desde el punto de vista formulaico? ¿Habrá diferencias si los evaluadores son nativos colombianos o españoles?

Se preparó un material que contenía una simulación de respuestas de examen de posicionamiento de nivel lingüístico que los docentes debían corregir. Fueron 8 textos que respondían a un mismo formato de prueba de competencia escrita con redacción de un correo electrónico a un profesor y su respuesta con las indicaciones para realizar la tutoría solicitada en una oficina externa al campus universitario. La distribución de estímulos en las pruebas se muestra en la Tabla 1.

Las direcciones son las contenidas en el ejercicio de Manual descripto en la sección 2 (Ainciburu et al., 2009, p. 37) y, por tanto, correctas en alguna variedad del español (Tabla 1, columna 3). Los encabezamientos de saludo en mensajes de correo electrónico provienen del corpus Nebrija-WOCAE (Rodríguez Velasco, Liu, 2019) y no se relacionan directamente con la variedad lingüística porque son de producción ELE. Estos corresponden al primer renglón realzado con el uso de dos puntos o coma antes del mensaje del texto. Estos dos tipos de reactivos se incluyeron en los textos de muestra que funcionaron como distractores en la corrección y que fueron reelaborados por los investigadores (Tabla 1, columna 4). Los errores que contenían, para estimular el macroobjetivo de la simulación, eran pocos y estrictamente gramaticales (uso de tiempos verbales y concordancia) y ortográficos (uso de mayúsculas), de modo que el posicionamiento general resultara en niveles avanzados de $\mathrm{C} 1$ y $\mathrm{C} 2$. 
Tabla 1. Reactivos utilizados para la calificación de adecuación de fenómenos pragmáticos

\begin{tabular}{|c|c|c|c|}
\hline Prueba & Saludo al profesor & Dirección propuesta & Variedad ELE \\
\hline 1. & $\begin{array}{l}\text { Estimado profesor } \\
\text { Rodriguez }\end{array}$ & $\begin{array}{l}\text { Av. Rivadavia 7145, P/B. Dpto. } 4 \\
1406 \text { Buenos Aires }\end{array}$ & Argentina \\
\hline 2. & Estimado Dr. Pedro & $\begin{array}{l}\text { Rohrmoser } 200 \text { metros norte del } \\
\text { Parque de la Amistad San José }\end{array}$ & Costa Rica \\
\hline 3. & Apreciado profesor Pedro & Carrera 7 No. 40 Bogotá D.C., & Colombia \\
\hline 4. & Estimado señor & $\begin{array}{l}\text { 3ra. y G, El Vedado } \\
\text { La Habana } 10400\end{array}$ & Cuba \\
\hline 5. & Hola profesor Pedro & $\begin{array}{l}\text { C/ Orense, } 22 \\
28020 \text { Madrid }\end{array}$ & España \\
\hline 6. & Estimado profesor & $\begin{array}{l}\text { Londres } 423 \text { Col. del Carmen } \\
\text { Coyoacán México, DF }\end{array}$ & México \\
\hline 7. & Profesor Rodríguez & $\begin{array}{l}\text { Jr. Talara } 307 \text { Comas - San Felipe, } \\
\text { Lima }\end{array}$ & Perú \\
\hline 8. & Hola Rodriguez & $\begin{array}{l}\text { Prudencio de Pena } 2544 \text { - } 11600 \\
\text { Montevideo }\end{array}$ & Uruguay \\
\hline
\end{tabular}

Actuaron como correctores de la prueba simulada 100 profesores ELE de Máster o Maestría en Lingüística Aplicada, en formación y con experiencia docente, 50 de la Universidad Javeriana de Bogotá (Colombia) y 50 de la Universidad Nebrija (España). En adelante se mencionarán como GPC y GPE (grupo de profesores colombianos y españoles, respectivamente).

La corrección suponía el uso de un baremo general de 4 posiciones con los constructos de Control ortográfico, Cohesión y coherencia discursiva y Adecuación pragmática al género. En este último criterio el baremo había sido adaptado a la tarea para obtener los datos de esta investigación (Tabla 2).

Tabla 2. Baremo analítico sobre la adecuación de fenómenos pragmáticos

\begin{tabular}{|c|l|}
\hline Puntaje & \multicolumn{1}{|c|}{ Descriptor } \\
\hline 4 & Se trata de una expresión nativa, perfectamente adecuada a la situación comunicativa. \\
\hline 3 & La expresión no tiene equivalente nativo, pero es cortés y se adapta al contexto. \\
\hline 2 & Es un uso nativo o cuasi nativo, que no parece adecuado a la situación comunicativa. \\
\hline 1 & El uso no semeja el de los nativos y no se adapta al contexto situacional. \\
\hline
\end{tabular}


El orden de las posiciones intermedias se basa en la constatación de que el uso de una expresión no nativa que logra su objetivo comunicativo y se adapta al contexto (3) es preferible a una que no es adecuada en la situación comunicativa, porque sus posibilidades de resultar perjudicial para la comprensión del mensaje son más altas (Alba Quiñones, 2009). Por esa razón, en el análisis de los resultados habrá que tener en cuenta que la graduación podría no comportarse exactamente como una escala de Likert a un solo factor.

\section{RESULTADOS Y DISCUSIÓN}

Se consideraron los 1600 juicios de adecuación a los reactivos (forma de saludo y direcciones) que se analizaron a la luz de los juicios de percepción nativa de la variedad lingüística del español utilizada. El resto de la prueba funcionó como contexto natural de la actividad y como distractor, aunque algún detalle de corrección pueda reforzar la hipótesis de trabajo.

En relación con el reactivo Dirección propuesta se solicitó que los docentes juzgaran de 1 a 4, con el mismo procedimiento ya usado para los saludos (Tabla 3 ).

Tabla 3. Percepción de la adecuación de "direcciones"

\begin{tabular}{|l|c|c|c|c|c|c|}
\hline \multicolumn{1}{|c|}{ Direcciones } & \multicolumn{3}{|c|}{ GPC (n= 50) } & \multicolumn{3}{c|}{ GPE (n=50) } \\
\hline $\begin{array}{l}\text { Av. Rivadavia 7145, P/B. Dpto. } \\
4 \quad \text { I406 Buenos Aires }\end{array}$ & 200 & 4 & 0 & 195 & 3,75 & 0,34 \\
\hline $\begin{array}{l}\text { Rohrmoser 200 metros norte } \\
\text { del Parque de la Amistad } \\
\text { San José }\end{array}$ & 94 & 1,88 & 0,25 & 86 & 1,72 & 0,44 \\
\hline Carrera 7 No. 40 Bogotá D.C. & 200 & 4 & 0 & 184 & 3,68 & 1,23 \\
\hline $\begin{array}{l}\text { 3ra. y G, El Vedado } \\
\text { La Habana 10400 }\end{array}$ & 96 & 1,92 & 0,42 & 90 & 1,8 & 0,12 \\
\hline $\begin{array}{l}\text { C/ Orense, 22 } \\
\text { 28020 Madrid }\end{array}$ & 200 & 4 & 0 & 200 & 4 & 0 \\
\hline $\begin{array}{l}\text { Londres 423 Col. del Carmen } \\
\text { Coyoacán México, DF }\end{array}$ & 195 & 3,75 & 0,34 & 197 & 3,94 & 0,22 \\
\hline $\begin{array}{l}\text { Jr. Talara 307 Comas - San } \\
\text { Felipe, Lima }\end{array}$ & 197 & 3,94 & 0,22 & 200 & 4 & 0 \\
\hline $\begin{array}{l}\text { Prudencio de Pena 2544 - } \\
\text { 11600 Montevideo }\end{array}$ & 200 & 4 & 0 & 200 & 4 & 0 \\
\hline
\end{tabular}


Dado que cada reactivo puede puntuarse con un valor máximo de 4 y mínimo de 1 y que los jueces por grupo son 50, el valor de referencia (V) mayor es 200 y el menor, 50. Los resultados muestran una situación menos variada respecto a la del reactivo "Saludos", en la que los docentes de ambos grupos polarizan los juicios. Los dos grupos de profesores consideran casi todas las direcciones adecuadas salvo la costarricense y la cubana. Para comprender los resultados, fue necesario que los investigadores realizaran un ejercicio donde se solicitó a los docentes que se leyeran las direcciones en voz alta. Todos reconocieron correctamente las abreviaturas "calle", "avenida", "distrito federal", "tercera" y "número". La forma "P/B" por "planta baja" o "piso bajo" resultó fácil de leer en su versión extendida, mientras que "departamento" por "apartamento" generó algunas dudas en el grupo de profesores españoles. La abreviatura "Col." en la dirección mexicana no siempre se leyó como "colonia" (solo el 25\%), sin que existiera diferencia en los dos grupos. La abreviatura que causó más problemas en los dos grupos fue "Jr." que mayoritariamente se leyó como "junior" ( $80 \%$ de la muestra). Al leerlo los docentes se dieron cuenta de que no podía tratarse de una abreviatura de nombre propio y se preocuparon por haber aceptado como correcta una abreviatura que no estaba bien o debía colocarse después del apellido "Talara". Solo un docente colombiano supo que la palabra abreviada era "jirón".

En cuanto a los resultados que dependen de la nacionalidad de los docentes, los informantes GPE no comprendían la forma "D.C" y aventuraron correctamente "distrito capital" (20\%) o "central" (20\%), leyendo mayoritariamente "di si" como si de inglés se tratara y sin leerlo en su forma extendida. Un buen número de GPE interpretó "Carrera" como apellido y solo en un segundo momento lo asoció a "calle", incluso comentando el parecido con el catalán.

En cuanto a la supuesta inadecuación de las direcciones cubana y costarricense, GPC y GPE no las consideran usos nativos porque son tan imprecisas que una carta no podría llegar a destino. Es curioso que las expresiones de un estudiante ELE se consideren erradas o poco precisas sin pensar que el estudiante pueda haberlas escuchado de boca de un nativo.

Respecto al Saludo al profesor del encabezado inicial de cartas simuladas por estudiantes ELE, los resultados de percepción de la adecuación se presentan en la Tabla 4.

Los valores de media (M) y desviación estándar (DE) obtenidos muestran que los dos grupos de participantes coinciden a la hora de considerar como apropiados los encabezamientos "Estimado profesor Rodríguez", "Estimado profesor", "Profesor Rodríguez" y "Estimado Dr. Pedro"; y como poco apropiados (con una media igual o menor que 2) "Hola profesor Pedro", "Estimado señor" y "Hola Rodríguez", que obtiene la puntuación más baja. Los dos grupos disienten en la consideración de "Apreciado profesor Pedro" (correcto para el grupo colombiano e incorrecto para el español). Los valores de desviación estándar contenidos muestran que los juicios son homogéneos dentro de cada grupo. 
Tabla 4. Percepción de la adecuación del "Saludo al profesor"

\begin{tabular}{|l|c|c|c|c|c|c|}
\hline \multicolumn{1}{|c|}{ Saludo al profesor } & \multicolumn{3}{c|}{ GPC (n= 50) } & \multicolumn{3}{c|}{ GPE (n=50) } \\
\hline & $\mathrm{V}$ & $\mathrm{M}$ & $\mathrm{DE}$ & $\mathrm{V}$ & $\mathrm{M}$ & $\mathrm{DE}$ \\
\hline Estimado profesor Rodríguez & 199 & 3,98 & 1,51 & 194 & 3,88 & 1,49 \\
\hline Estimado Dr. Pedro & 149 & 2,98 & 1,02 & 167 & 3,34 & 0,94 \\
\hline Apreciado profesor Pedro & 187 & 3,74 & 1,81 & 94 & 1,88 & 0,34 \\
\hline Estimado señor & 96 & 1,92 & 0,42 & 91 & 1,82 & 1,03 \\
\hline Hola profesor Pedro & 71 & 1,42 & 0,89 & 83 & 1,66 & 0,24 \\
\hline Estimado profesor & 197 & 3,94 & 1,74 & 199 & 3,98 & 1,32 \\
\hline Profesor Rodríguez & 125 & 2,5 & 1,62 & 117 & 2,34 & 1,02 \\
\hline Hola Rodríguez & 59 & 1,18 & 0,18 & 51 & 1,02 & 0,23 \\
\hline
\end{tabular}

En una primera consideración de tipo cualitativa verificamos que los encabezados que los GPC y los GPE estimaron más formales suelen puntuar más alto que los informales, que se juzgan poco adecuados (en forma notoria los que contienen "Hola"). La situación resulta diversificada en el caso de combinatorias como "Estimado señor" que requieren una indagatoria más fina. Es posible que haya que indagar mucho más porque la articulación de la fórmula tomada como referencia (Economidou-Kogetsidis, 2011; Níkleva \& Núñez Delgado, 2013; Codina Espurz \& Salazar Campillo, 2019) no se muestra poco predictiva respecto a su adecuación. La indagación acerca de lo que los profesores evaluadores creen saber sobre la adecuación debería ampliarse (Andión Herrera, 2019), sobre todo a los profesores no nativos que podrían ser más sensibles en este sentido. La nuestra es una aportación original en este sentido y se produce en un área en la que muchos afirman que hay continuidad, porque la variación geolectal en el español culto debería ser menor al de uso cotidiano.

$\mathrm{Si}$ bien hemos tenido que recurrir a comentarios y no solo a los juicios para aclarar la comprensión de las direcciones por parte de los docentes, los resultados muestran que los evaluadores nativos desconocen algunas formas de expresar direcciones propias de la lengua española. En este sentido, los resultados cuantitativos que interesan a lo que en la Tabla 1 llamamos Variedad $\boldsymbol{E L E}$ ven a las variedades cubana y costarricense como las menos conocidas por los docentes, pero el ejercicio de lectura posterior muestra que otras, como la peruana o la mexicana, podrían considerarse "poco conocidas". Cuando los resultados que se examinan tienen que ver con el grupo español, la variedad colombiana también resulta poco conocida. Claramente las direcciones pueden considerarse solo una espía del desconocimiento acerca de cómo se expresan en diferentes variedades nativas, pero justamente el hecho de que se trate de una función de nivel básico (A2) debería alarmar en tiempos de declarado panhispanismo. 


\section{CONCLUSIONES}

En este estudio se eligieron dos tipos de expresiones que implican un conocimiento pragmático ligado a las culturas académicas (los saludos) y a la localización. Ambos reactivos suponen un conocimiento de las variedades del español por parte de los profesores que actúan como jueces en la simulación de corrección de un examen de certificación ELE. Los resultados cuantitativos de los grupos de colombianos (GPC) y españoles (GPE) reportados en el apartado previo muestran un panorama de juicios focalizados de adecuación pragmática de los reactivos muy compacto. Sin embargo, aunque los juicios disidentes representen el factor más sorprendente desde el punto de vista de la interculturalidad, creemos que un análisis cualitativo de los comportamientos observados, ligado a la investigación previa, ayuda a comprender en detalle los resultados en su globalidad y a formular hipótesis de mayor complejidad en relación con los presupuestos que guían el comportamiento de los docentes en su condición de evaluadores.

Los resultados muestran que los profesores nativos tienden a considerar como correctos los estímulos que conocen porque son de uso en su propia variedad (como el de Apreciado o Carrera en el caso de los colombianos) o se presentan con el mismo formato de los manuales ELE mayormente utilizados. Paralelamente, tanto los profesores nativos colombianos como los españoles tienden a puntuar muy bajo o como error inter- o intralingüístico las diferentes variaciones geolectales del español. Aunque este fenómeno deba ser indagado con grupos de profesionales de más de dos proveniencias, estos datos sugieren que existe un efecto potencialmente "educativo" de los manuales ELE o de la variedad peninsular que representan. Los profesores españoles no reconocieron la tipicidad de fórmulas americanas que no están en los libros, los profesores colombianos tampoco, a menos que se tratara de su propia variedad. Es muy posible que sea deseable una presencia mayor de realia en los manuales ELE por razones de una mayor exposición al input, pero nos parece urgente que haya una mayor representación de las variedades lingüísticas. Si no se redujese a los geosinónimos, como en el citado ejemplo de camión, guagua, micro, autobús y se extendiera a fórmulas más amplias como se muestra en los ejemplos usados en la investigación primaria de este trabajo, sería aún mejor, puesto que la representatividad de las variantes no puede relegarse a la dimensión "palabra".

Creemos que a la luz de los resultados hay dos preguntas a las que debería dirigirse posteriormente la investigación: ¿la adecuación es un ámbito donde realmente los profesores nativos tienen ventaja como evaluadores? Es posible que no sea así y, en segundo orden, ¿cuánto se ganaría con la publicación pluricéntrica de manuales ELE? 


\section{BIBLIOGRAFÍA}

Ainciburu, M.C. (2018). El estudio de los géneros académicos en la producción textual en ELE. Journal of Spanish Language Teaching, 5 (2), 139-151. DOI: 10.1080/23247797.2018.1538341.

Ainciburu, M.C., González Rodríguez, V., Navas Méndez, A., Tayefeh, E., Vázquez, G. (2009). Con dinámica. Competencias y estrategias. Stuttgart: Klett.

Ainciburu, M.C., Méndez, A.N., Rodríguez, V.G., Tayefeh, E., Vázquez, G.E. (2011). Vía rápida: curso intensivo de español. Barcelona: Difusión.

de Alba Quiñones, V. (2009). El análisis de errores en el campo del español como lengua extranjera: algunas cuestiones metodológicas. Revista Nebrija de Lingüistica aplicada a la enseñanza de Lenguas, 3 (5), 1-16. DOI: 10.26378/rnlael35103.

Andión Herrero, M.A. (2019). La unidad y variedad del español en el marco glotopolítico y aplicado actual. Journal of Spanish Language Teaching, 6 (2), 150-169. DOI: 10.1080/23247797.2019.1681627.

Brown, P., Levinson, S. (1978). Universals in language usage: Politeness phenomena. In E. Goody (Ed.), Questions and politeness (pp. 56-89). Cambridge: Cambridge University Press.

Codina Espurz, V., Salazar Campillo, P. (2019). Openings and Closings in Emails by CLIL Students: A Pedagogical Proposal. English Language Teaching, 12 (2), 57-67. DOI: 10.5539/elt.v12n2p57.

Economidou-Kogetsidis, M. (2011). "Please answer me as soon as possible": Pragmatic failure in non-native speakers' e-mail requests to faculty. Journal of Pragmatics, 43 (13), 3193-3215. DOI: 10.1016/j. pragma.2011.06.006.

de la Fuente Cobas, I., Wei, N. (2020). Profesores nativos y no nativos de español: situación y desarrollo de la colaboración docente en las universidades chinas. Journal of Spanish Language Teaching, 1-17. DOI: $10.1080 / 23247797.2020 .1770960$.

Gavarrete, M., Albanese, V. (2018). Propuesta de aula para abordar la ubicación espacial y el plano cartesiano desde la interculturalidad. Uno-Revista de Didáctica de las Matemáticas, 82, 23-30. Recuperado de: https://www.researchgate.net/publication/333479991.

Robles Garrote, P. (2017). Perspectivas de análisis pragmático de correos electrónicos de petición. Estudio contrastivo en italiano y español L1/L2 (tesis doctoral inédita). Madrid: Universidad Antonio de Nebrija.

Rodríguez Velasco, D., Liu, X. (2019). Corpus Nebrija-WOCAE (Written and Oral Chinese Academic Emails). Disponible en: https://slabank.talkbank.org/access/Spanish/Nebrija-WOCAE.html 
ANEXO 1. ACTIVIDAD DE MANUAL (Ainciburu et al., 2009)

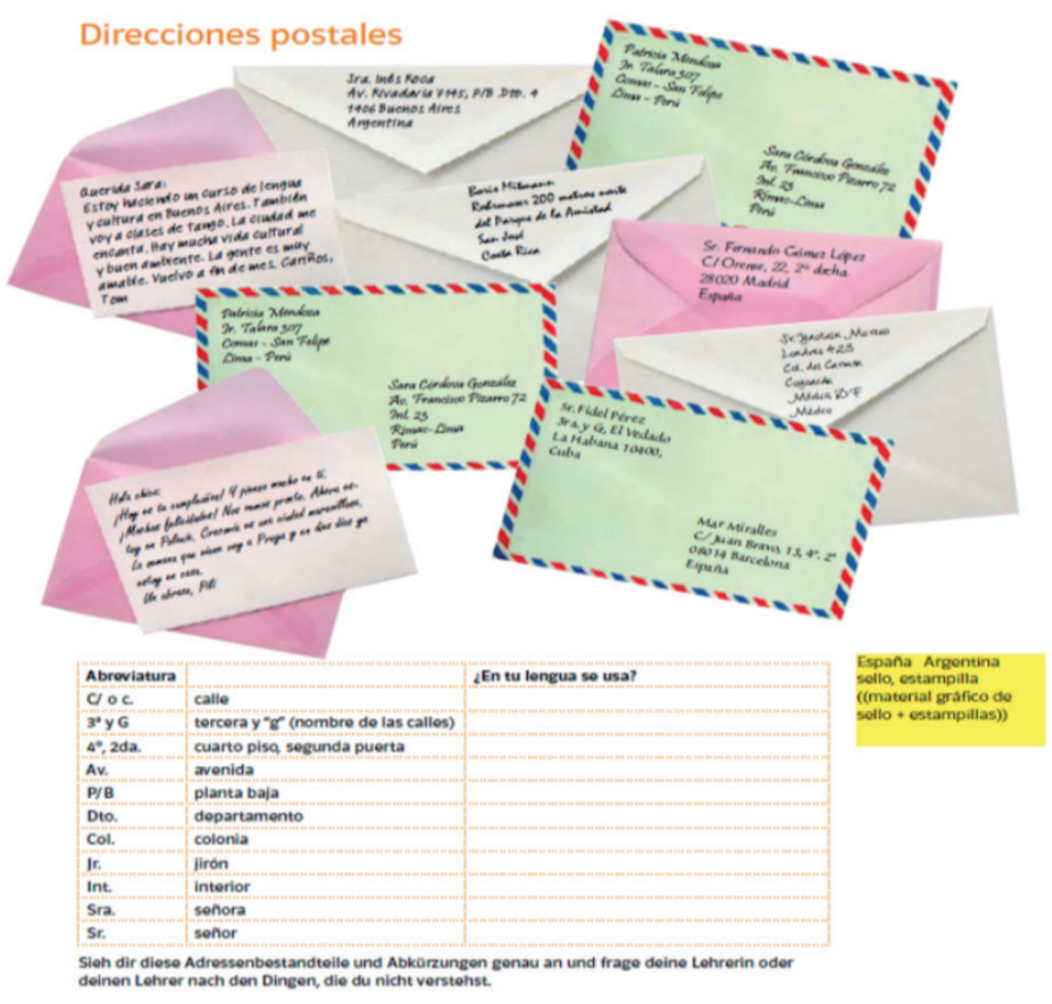

